Proyecciones Journal of Mathematics

Vol. 31, No 3, pp. 261-275, September 2012.

Universidad Católica del Norte

Antofagasta - Chile

\title{
Symmetric regular cacti-properties and enumeration
}

\author{
S. K. VAIDYA \\ Saurashtra University, India \\ and \\ D. D. Bantva \\ L. E. College, India \\ Received: March 2012. Accepted : June 2012
}

\begin{abstract}
A cactus is a connected graph whose all the blocks are isomorphic to cycle or complete graph on $n$ vertices. We introduce symmetric regular cacti and a procedure for their construction. We discuss some characteristics of symmetric regular cacti. The number of symmetric regular cacti on given number of vertices are also enumerated. A possible application of present work to a real world problem for the committee formation is also suggested.
\end{abstract}

Keywords : Block, cactus, cut vertex.

AMS Subject Classification Number : 05C75, 05C30, 05C90. 


\section{Introduction}

We begin with the definition of a graph which is a triple consisting of a vertex set $V(G)$, an edge set $E(G)$, and a relation that associates each edge with two vertices called its endpoints. The edge $e$ having identical end vertices is called a loop and two distinct edges with same end vertices are called parallel edges. A graph is called simple if it has neither loops nor parallel edges. A graph $G$ is called bipartite graph if the vertex set $V(G)$ can be partitioned into two nonempty subsets $X$ and $Y$ in such a way that each edge of $G$ has one end vertex in $X$ and other end vertex in $Y$. A complete graph $K_{n}$ is a simple graph in which each pair of distinct vertices is joined by an edge. A walk in a graph $G$ is a finite sequence $W=$ $v_{0} e_{1} v_{1} e_{2} v_{2} \ldots v_{k-1} e_{k} v_{k}$ whose terms are alternately vertices and edges such that, for $1 \leq i \leq k$, the edge $e_{i}$ has ends $v_{i-1}$ and $v_{i}$. A path $P_{n}$ is a walk in which vertices are distinct. A path in which $v_{0}=v_{k}$ is called a cycle denoted by $C_{n}$. A graph without any cycle is called acyclic graph. A vertex $u$ is said to be connected to a vertex $v$ in a graph $G$ if there is a path in $G$ from $u$ to $v$ and a graph $G$ is called connected if every two of its vertices are connected otherwise it is called disconnected. If $G$ has an $u, v$-path, then the distance from $u$ to $v$, written $d(u, v)$, is the least length of a $u, v$-path. The diameter of $G$ denoted by $\operatorname{diam} G$ is $\max \{d(u, v): u, v \in V(G)\}$. The eccentricity of the vertex $u$, written as $\epsilon(u)$, is $\max \{d(u, v): v \in V(G)\}$. The radius of a graph $G$, written as $\operatorname{rad} G$, is $\min \{\epsilon(u): u \in V(G)\}$. The center of a graph $G$ is the subgraph induced by the vertices of minimum eccentricity. A vertex $v$ of a graph $G$ is called a cut vertex if its deletion leaves a graph disconnected. A block of a graph $G$ is a maximal connected subgraph of $G$ that has no cut-vertices.

Definition 1.1. An $n$-complete cactus $C\left(K_{n}\right)$ is a simple graph whose all the blocks are isomorphic to $K_{n}$.

Definition 1.2. An $n$-complete $k$-regular cactus $C\left(K_{n}(k)\right)$ is an $n$-complete cactus in which each cut vertex is exactly in $k$ blocks.

For $n$-complete $k$-regular cactus, readers should not be confused with the word regularity as the regularity is not in the sense of degree but it is the total number of blocks in which the given cut vertex lies. The block which contains only one cut vertex is called leaf block and that cut vertex is known as leaf block cut vertex. In $n$-complete $k$-regular cactus the vertices which are not cut vertices are known as leaf vertices. 
Definition 1.3. A symmetric $n$-complete $k$-regular cactus $S C\left(K_{n}(k)\right)$ is an $n$-complete $k$-regular cactus in which the eccentricity of each leaf vertex is the same.

Some people defines the cactus with blocks isomorphic to cycle $C_{n}$ which is known as $n$-ary cactus $[1,2]$ but we have considered here $K_{n}$ instead of $C_{n}$ in more general sense. Throughout this discussion by symmetric regular cactus we mean symmetric $n$-complete $k$-regular cactus with $p$ vertices, $b$ blocks and $q$ edges. Sometimes we use notation $S C\left(K_{n}(k)\right)(d)$ for symmetric $n$-complete $k$-regular cactus of diameter $d$. A single block is recognize as a trivial cactus is of no importance for us as it is isomorphic to $K_{n}$ and will satisfy all the properties of $K_{n}$. For all other standard terminology and notations we refer to West[3].

\section{Construction of Symmetric Regular Cactus}

We describe the procedure to construct symmetric regular cactus of odd and even diameter.

\subsection{Symmetric regular cactus of odd diameter:}

Let $K_{n}$ be the complete graph on $n$ vertices say $v_{1}, v_{2}, \ldots, v_{n}$ which is a trivial cactus. Take more $n(k-1)$ copies of $K_{n}$. For $1 \leq i \leq n$, identify one vertex of each copy of $K_{n}$ from a bunch of $(k-1)$ copies of $K_{n}$ with each $v_{i}$. Then the resultant graph is a symmetric $n$-complete $k$-regular cactus with diameter three. In this symmetric $n$-complete $k$-regular cactus, the vertices $v_{1}, v_{2}, \ldots, v_{n}$ are now cut vertices and all other vertices are leaf vertices. Continuing this process, we can construct a symmetric regular cactus of odd diameter.

\subsection{Symmetric regular cactus of even diameter:}

Let $v_{0}$ be any vertex. Take $k$ copy of $K_{n}$ and identify one vertex of each $K_{n}$ with $v_{0}$. Then it is symmetric $n$-complete $k$-regular cactus of diameter two. Here $v_{0}$ is a cut vertex and all other vertices are leaf vertices. Now to construct desired symmetric $n$-complete $k$-regular cactus with even diameter follow the procedure as we have described in section 2.1 for symmetric 
regular cactus of required even diameter.

We note that the center of a symmetric regular cactus is either a vertex or a block and accordingly it is called block centered symmetric regular cactus or vertex center symmetric regular cactus. If diameter is odd then it is block centered and if diameter is even then it is vertex centered. In a symmetric $n$-complete $k$-regular cactus $S C\left(K_{n}(k)\right)$, a vertex $v$ is a terminal or leaf vertex if $d(v)=n-1$ and the block which contains a terminal vertex is called terminal or leaf block. Also a vertex which is not leaf vertex is known as cut vertex or internal vertex and a block which is not leaf block is known as internal block.

Example 2.1. A 4-complete cactus and a symmetric 4-complete 2-regular cactus of diameter 5 and 6 are shown in Figure 1 and Figure 2 respectively.

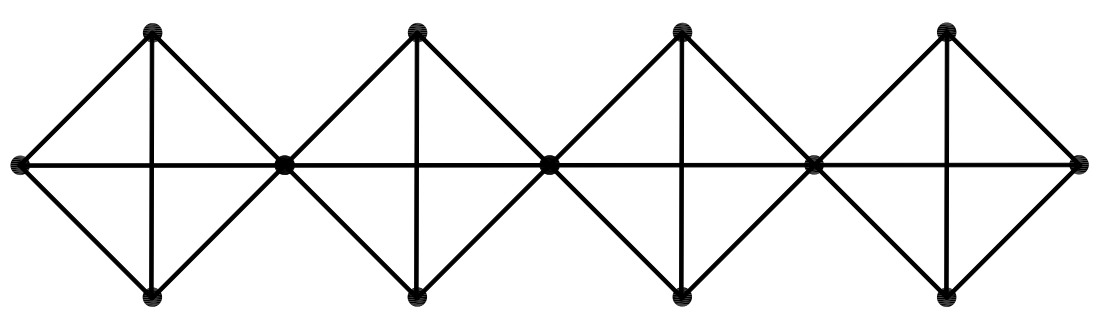

Figure 1 : 4-complete cactus.
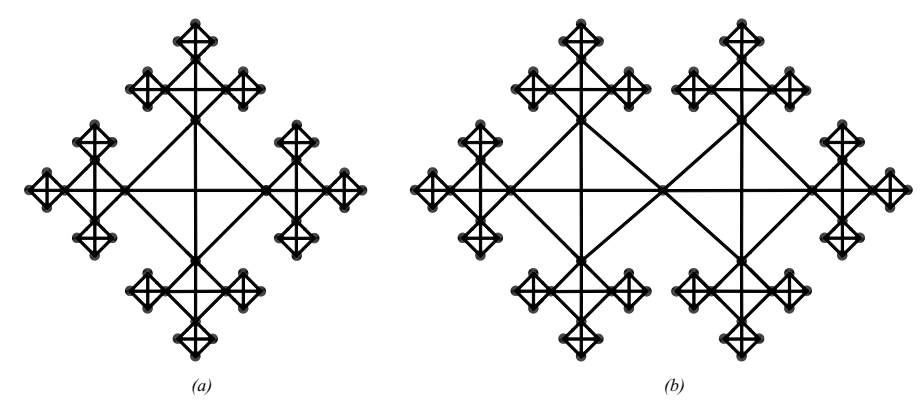

Figure 2 : Symmetric 4-complete 2-regular cactus of diameter 5 and 6. 
Observation 2.2. From the definition and construction of non trivial symmetric regular cactus, it is clear that the

- $2 \leq n \leq p$

- $2 \leq d \leq p-1$

- $2 \leq k \leq p-1$

\section{Properties of Symmetric Regular Cactus}

Theorem 3.1. For any $S C\left(K_{n}(k)\right)$ of diameter $d$,

$p=\left\{\begin{array}{l}n+\sum_{i=1}^{\frac{d-1}{2}} n(k-1)^{i}(n-1)^{i} ; \quad \text { if } \quad d \quad \text { is odd } \\ 1+\sum_{i=1}^{\frac{d}{2}} k(k-1)^{i-1}(n-1)^{i} ; \quad \text { if } \quad d \quad \text { is even }\end{array}\right.$

$b=\left\{\begin{array}{l}1+\sum_{i=1}^{\frac{d-1}{2}} n(k-1)^{i}(n-1)^{i-1} ; \quad \text { if } \quad d \quad \text { is odd } \\ k+\sum_{i=1}^{\frac{d}{2}-1} k(k-1)^{i}(n-1)^{i} ; \quad \text { if } \quad d \quad \text { is even }\end{array}\right.$

Moreover, in the formula of $p$ and $b$, the last term of the summation gives the total number of leaf vertices and leaf blocks respectively while the sum of remaining terms gives the total number of cut vertices and internal blocks respectively.

Proof: We prove this theorem using induction on $d$. If $d=1$ then cactus is just one block which has $n$ leaf vertices as each vertex is of degree $n-1$. If $d=2$ then regular cactus is a vertex centered and it is $k$ copies of complete graph $K_{n}$ each one sharing a common vertex. Therefore the number of vertices $p=k n-k+1=1+k(n-1)$ and the common vertex is a cut vertex and the regular cactus has $k(n-1)$ leaf vertices while the number of blocks is $k$.

Let the result is true for $d=t$ then 
Case-1: If $t$ is odd then it has $n+\sum_{i=1}^{\frac{t-1}{2}} n(k-1)^{i}(n-1)^{i}$ vertices and $1+\sum_{i=1}^{\frac{d-1}{2}} n(k-1)^{i}(n-1)^{i-1}$ blocks. In the formula of $p$ and $b$, the last term of the summation which are $n(k-1)^{\frac{t-1}{2}}(n-1)^{\frac{t-1}{2}}$ and $n(k-1)^{\frac{t-1}{2}}(n-1)^{\frac{t-3}{2}}$ gives the total number of leaf vertices and leaf blocks respectively while the sum of remaining terms gives the total number of cut vertices and internal blocks respectively. Now again from a bunch of $k-1$ copies of $K_{n}$ identify the one vertex of each copy of $K_{n}$ with each leaf vertex then the total number of vertices $p$

$$
\begin{aligned}
& =n+\sum_{i=1}^{\frac{t-1}{2}} n(k-1)^{i}(n-1)^{i}+n(k-1)^{\frac{t-1}{2}}(k-1)(n-1)^{\frac{t-1}{2}}(n-1) \\
= & n+\sum_{i=1}^{\frac{t+1}{2}} n(k-1)^{i}(n-1)^{i} ;
\end{aligned}
$$

the total number of blocks $b$

$$
\begin{aligned}
& =1+\sum_{i=1}^{\frac{t-1}{2}} n(k-1)^{i}(n-1)^{i-1}+n(k-1)^{\frac{t-1}{2}}(k-1)(n-1)^{\frac{t-3}{2}}(n-1) \\
& =1+\sum_{i=1}^{\frac{t+1}{2}} n(k-1)^{i}(n-1)^{i-1} .
\end{aligned}
$$

Case-2: If $t$ is even then it has $1+\sum_{i=1}^{\frac{t}{2}} k(k-1)^{i-1}(n-1)^{i}$ vertices and $k+\sum_{i=1}^{\frac{t}{2}-1} k(k-1)^{i}(n-1)^{i}$ blocks. In the formula of $p$ and $b$, the last term of the summation which are $k(k-1)^{\frac{t}{2}-1}(n-1)^{\frac{t}{2}}$ and $k(k-1)^{\frac{t}{2}-1}(n-1)^{\frac{t}{2}-1}$ gives the total number of leaf vertices and leaf blocks respectively while the sum of remaining terms gives the total number of cut vertices and internal blocks respectively. Now again from a bunch of $k-1$ copies of $K_{n}$ identify a vertex of each copy of $K_{n}$ with each leaf vertex then the total number of vertices $p$

$$
=1+\sum_{i=1}^{\frac{t}{2}} k(k-1)^{i-1}(n-1)^{i}+k(k-1)^{\frac{t}{2}-1}(k-1)(n-1)^{\frac{t}{2}}(n-1)
$$


$=1+\sum_{i=1}^{\frac{t}{2}+1} k(k-1)^{i-1}(n-1)^{i}$

the total number of blocks $b$

$=k+\sum_{i=1}^{\frac{t}{2}-1} k(k-1)^{i}(n-1)^{i}+k(k-1)^{\frac{t}{2}-1}(k-1)(n-1)^{\frac{t}{2}-1}(n-1)$

$=1+\sum_{i=1}^{\frac{t}{2}} k(k-1)^{i}(n-1)^{i}$.

Hence, the result is true for $d=t+k$. Therefore, the result is true for all $d$.

Observation 3.2. For symmetric regular cactus on $p$ vertices, the trivial cactus $K_{n}$ gives minimum value of $d=1$ and a path on $p$ vertices gives the maximum value of $d=p-1$.

Theorem 3.3. If $S C\left(K_{n}(k)\right)$ is symmetric regular cactus with $p$ vertices then it has precisely $\frac{1}{2} n(p-1)$ edges.

Proof: Let $S C\left(K_{n}(k)\right)$ be a symmetric regular cactus with $p$ vertices then it has $1+\sum_{i=1}^{\frac{d-1}{2}} n(k-1)^{i}(n-1)^{i-1}$ blocks if $d$ is odd and $k+\sum_{i=1}^{\frac{d}{2}-1} k(k-1)^{i}(n-1)^{i}$ blocks if $d$ is even. Now as each block is isomorphic to $K_{n}$ it has $\frac{1}{2} n(n-1)$ edges. Therefore the total number of edges $q$

$$
\begin{aligned}
& =\left\{\begin{array}{l}
\left\{1+\sum_{i=1}^{\frac{d-1}{2}} n(k-1)^{i}(n-1)^{i-1}\right\} \frac{1}{2} n(n-1) ; \quad \text { if } \quad d \quad \text { is odd } \\
\left\{k+\sum_{i=1}^{\frac{d}{2}-1} k(k-1)^{i}(n-1)^{i}\right\} \frac{1}{2} n(n-1) ; \quad \text { if } \quad d \quad \text { is even }
\end{array}\right. \\
& =\left\{\begin{array}{l}
\frac{1}{2} n\left\{(n-1)+\sum_{i=1}^{\frac{d-1}{2}} n(k-1)^{i}(n-1)^{i}\right\} ; \quad \text { if } \quad d \quad \text { is odd } \\
\frac{1}{2} n\left\{k(n-1)+\sum_{i=2}^{\frac{d}{2}} k(k-1)^{i-1}(n-1)^{i}\right\} ; \quad \text { if } \quad d \quad \text { is even }
\end{array}\right.
\end{aligned}
$$




$$
=\left\{\begin{array}{l}
\frac{1}{2} n\left\{n+\sum_{i=1}^{\frac{d-1}{2}} n(k-1)^{i}(n-1)^{i}-1\right\} ; \quad \text { if } \quad d \quad \text { is odd } \\
\frac{1}{2} n\left\{1+\sum_{i=1}^{\frac{d}{2}} k(k-1)^{i-1}(n-1)^{i}-1\right\} ; \quad \text { if } \quad d \quad \text { is even }
\end{array}\right.
$$

$=\frac{1}{2} n(p-1)$. Hence the result.

Corollary 3.4. There can not exist symmetric regular cacti on even number of vertices with odd completeness of blocks.

Proof: We prove by contradiction. Suppose that there exists a symmetric regular cactus on even number of vertices with odd completeness of blocks then $p$ is even and $n$ is odd and hence $\frac{1}{2} n(p-1)$ is not integer. But by Theorem 3.3, the total number of edges in a symmetric regular cactus is $q$ $=\frac{1}{2} n(p-1)$ which is not an integer, a contradiction. Hence the result.

Theorem 3.5. A symmetric $n$-complete $k$-regular cactus is Eulerian if and only if $n$ is odd.

Proof: We use the fundamental result that a connected graph $G$ is Eulerian if and only if each vertex in $G$ has even degree. The degree of each vertex in symmetric $n$-complete $k$-regular cactus is even if and only if $n$ is odd and hence the result.

Observation 3.6. A symmetric $n$-ary $k$-regular cactus is always Eulerian.

\section{Enumeration of Symmetric Regular Cactus}

In this section, we enumerate all symmetric regular cactus on given $p$ vertices as follows.

Theorem 4.1. The total number of symmetric $n$-complete $k$-regular cactus of diameter $d$ on $p$ vertices is the total number of positive integral solutions of equations

$n\left(\Phi_{\frac{d+1}{2}}((n-1)(k-1))=p\right.$ for odd $d$ and $1+k(n-1) \Phi_{\frac{d}{2}}((n-1)(k-1))=p$ for even $d$, 
where $\Phi_{p}(x)=1+x+x^{2}+\ldots+x^{p-1}$.

Proof: Let $S C\left(K_{n}(k)\right)(d)$ be the symmetric $n$-complete $k$-regular on $p$ vertices and diameter $d$. Then by Theorem 3.1, the positive integers $n, k$ and $d$ satisfy the equations

$p=n+\sum_{i=1}^{\frac{d-1}{2}} n(k-1)^{i}(n-1)^{i}$ if $d$ is odd and $p=1+\sum_{i=1}^{\frac{d}{2}} k(k-1)^{i-1}(n-1)^{i}$ if $d$ is even.

i.e $n\left(\Phi_{\frac{d+1}{2}}((n-1)(k-1))=p\right.$ if $d$ is odd and $1+k(n-1) \Phi_{\frac{d}{2}}((n-1)(k-1))=p$ if $d$ is even, where $\Phi_{p}(x)=1+x+x^{2}+\ldots+x^{p-1}$.

On the other hand, for $p$ there exist positive integers $n, k$ and $d$ which satisfy the equations $n\left(\Phi_{\frac{d+1}{2}}((n-1)(k-1))=p\right.$ for odd $d$ and $1+k(n-$ 1) $\Phi_{\frac{d}{2}}((n-1)(k-1))=p$ for even $d$, where $\Phi_{p}(x)=1+x+x^{2}+\ldots+x^{p-1}$. Now, construct symmetric $n$-complete $k$-regular cactus on given number of $p$ vertices by employing the construction procedure described in sections 2.1 and 2.2 respectively.

Corollary 4.2. A trivial cactus is the only symmetric regular cactus of odd diameter on prime number of vertices.

Proof: Suppose that there exists a nontrivial symmetric regular cactus, where $d$ odd, $p$ is prime and $3 \leq d \leq p-1,1<n<p$ and $1<k<p-1$ such that

$n\left(\Phi_{\frac{d+1}{2}}((n-1)(k-1))\right)=p$,

i.e. $n+n(k-1)(n-1)+n(k-1)^{2}(n-1)^{2}+\ldots+n(k-1)^{\frac{d-1}{2}}(n-1)^{\frac{d-1}{2}}=p$

i.e. $n\left(1+(k-1)(n-1)+(k-1)^{2}(n-1)^{2}+\ldots+(k-1)^{\frac{d-1}{2}}(n-1)^{\frac{d-1}{2}}\right)=p$

i.e. $n \mid p$ which contradicts the fact that $p$ is prime. Hence the result.

Corollary 4.3. $S C\left(K_{2}(p)\right)$ is the only symmetric regular cactus of even diameter on $p+1$ vertices, where $p$ is prime.

Proof: Suppose that there exists a symmetric regular cactus other than $S C\left(K_{2}(p)\right)$ with $d$ is even and $2<d \leq p-1,2<n \leq p$ and $1<k<p$ such that 
$1+k(n-1) \Phi_{\frac{d}{2}}((n-1)(k-1))=p+1$

i.e. $1+k(n-1)+k(k-1)(n-1)^{2}+k(k-1)^{2}(n-1)^{3}+\ldots+k(k-1)^{\frac{d}{2}-1}(n-1)^{\frac{d}{2}}=$ $p+1$.

i.e. $k(n-1)\left[1+(k-1)(n-1)+(k-1)^{2}(n-1)^{2}+\ldots+(k-1)^{\frac{d}{2}-1}(n-1)^{\frac{d}{2}-1}\right]=p$.

i.e. $k \mid p$ which contradicts the fact that $p$ is prime. Hence the result.

Example 4.4. In the following Table-1 all symmetric regular cacti with number of vertices $\leq 10$ is given. 


\begin{tabular}{|c|c|c|c|c|c|}
\hline $\begin{array}{c}\text { Vertices } \\
p\end{array}$ & $\begin{array}{l}\text { Total No. } \\
\text { of cactus }\end{array}$ & $\begin{array}{l}\text { Cactus } \\
\text { Type }\end{array}$ & $\begin{array}{c}\text { Diameter } \\
d\end{array}$ & $\begin{array}{c}\text { Regularity } \\
k\end{array}$ & $\begin{array}{c}\text { Completeness } \\
n\end{array}$ \\
\hline 1 & 1 & Odd $=1$ & 0 & 1 & 1 \\
\hline 2 & 1 & Odd $=1$ & 1 & 1 & 2 \\
\hline \multirow[t]{2}{*}{3} & \multirow[t]{2}{*}{2} & Odd $=1$ & 1 & 1 & 3 \\
\hline & & Even $=1$ & 2 & 2 & 2 \\
\hline \multirow[t]{3}{*}{4} & \multirow[t]{3}{*}{3} & \multirow[t]{2}{*}{ Odd $=2$} & 1 & 1 & 4 \\
\hline & & & 3 & 2 & 2 \\
\hline & & Even $=1$ & 2 & 3 & 2 \\
\hline \multirow[t]{4}{*}{5} & \multirow[t]{4}{*}{4} & Odd $=1$ & 1 & 1 & 5 \\
\hline & & \multirow[t]{3}{*}{ Even $=3$} & 2 & 2 & 3 \\
\hline & & & 2 & 4 & 2 \\
\hline & & & 4 & 2 & 2 \\
\hline \multirow[t]{4}{*}{6} & \multirow[t]{4}{*}{$\overline{\overline{4}}$} & \multirow[t]{3}{*}{$\bar{O}$ Odd $=3$} & 1 & 1 & 6 \\
\hline & & & 3 & 3 & 2 \\
\hline & & & 5 & 2 & 2 \\
\hline & & Even $=1$ & 2 & 5 & 2 \\
\hline \multirow[t]{5}{*}{7} & \multirow[t]{5}{*}{5} & Odd $=1$ & 1 & 1 & 7 \\
\hline & & \multirow[t]{4}{*}{ Even $=4$} & 2 & 2 & 4 \\
\hline & & & 2 & 3 & 3 \\
\hline & & & 2 & 6 & 2 \\
\hline & & & 6 & 2 & 2 \\
\hline \multirow[t]{4}{*}{8} & \multirow[t]{4}{*}{4} & \multirow[t]{3}{*}{$\operatorname{Odd}=3$} & 1 & 1 & 8 \\
\hline & & & 3 & 4 & 2 \\
\hline & & & 7 & 2 & 2 \\
\hline & & Even $=1$ & 2 & 7 & 2 \\
\hline \multirow[t]{6}{*}{9} & \multirow[t]{6}{*}{6} & \multirow[t]{2}{*}{$\operatorname{Odd}=2$} & 1 & 1 & 9 \\
\hline & & & 3 & 2 & 3 \\
\hline & & \multirow[t]{4}{*}{ Even $=4$} & 2 & 2 & 5 \\
\hline & & & 2 & 4 & 3 \\
\hline & & & 2 & 8 & 2 \\
\hline & & & 8 & 2 & 2 \\
\hline \multirow[t]{6}{*}{10} & \multirow[t]{6}{*}{6} & \multirow[t]{3}{*}{ Odd $=3$} & 1 & 1 & 10 \\
\hline & & & 3 & 5 & 2 \\
\hline & & & 9 & 2 & 2 \\
\hline & & \multirow[t]{3}{*}{ Even $=3$} & 2 & 3 & 4 \\
\hline & & & 2 & 9 & 2 \\
\hline & & & 4 & 3 & 2 \\
\hline
\end{tabular}




\section{Drawing of Symmetric Regular Cactus}

For more clarity of the concept, all the symmetric regular cacti on 9 vertices are given.

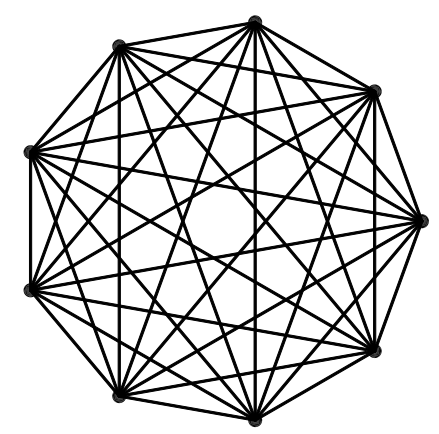

Figure 3 : Trivial Cactus on 9 vertices.

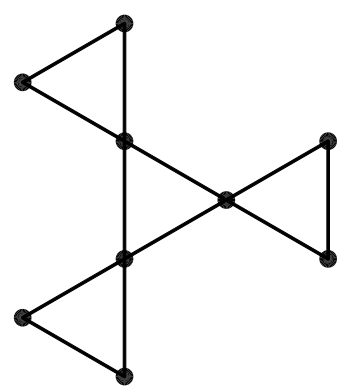

Figure 4 : Symmetric 3-complete 2-regular cactus of diameter 3.

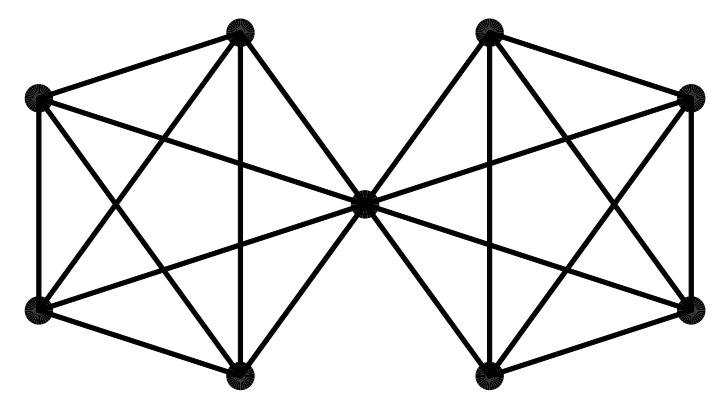

Figure 5 : Symmetric 5-complete 2-regular cactus of diameter 2. 


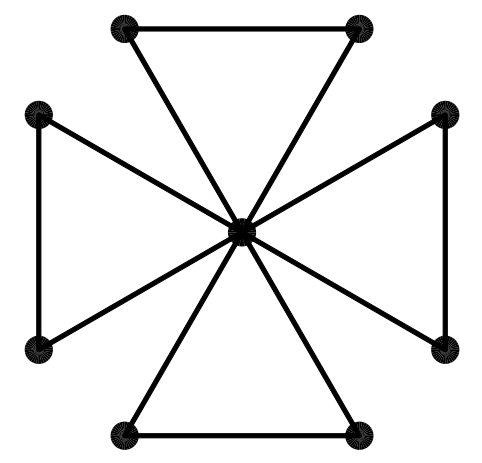

Figure 6 : Symmetric 3-complete 4-regular cactus of diameter 2.

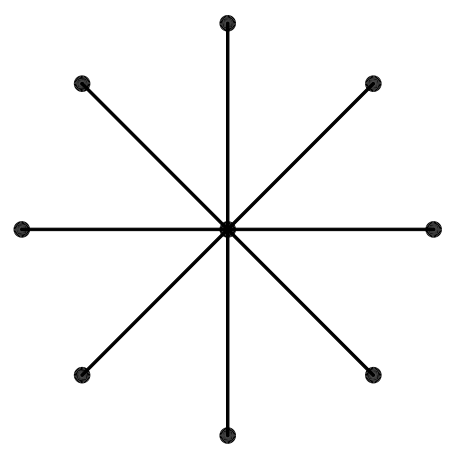

Figure 7 : Symmetric 2-complete 8-regular cactus of diameter 2.

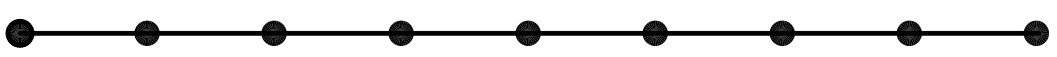

Figure 8 : Symmetric 2-complete 2-regular cactus of diameter 8. 


\section{Application}

In this section, we discuss an application of symmetric regular cactus. The formation of committees in any adminstration often gives rise to a symmetric regular cactus provided the number of persons in each committee is equal to say $n$. Then a single person or a committee which is formed for any purpose will can form other committees under them. Any member(s) of committee pass over a specific task to other committee under own leadership. Such arrangement will create either vertex centered symmetric regular cactus or block centered symmetric regular cactus. Again each member of this committee make other committees to make their work easy. Continuing in this way the formation of committees will give rise to a symmetric regular cactus. The main advantage of such model is that the work is evenly distributed and the whole system will work effectively. This model is more fruitful only if the graph remains connected.

\section{Concluding Remarks}

We have introduced a concept of symmetric $n$-complete $k$-regular cacti and also obtain the exact number of vertices, blocks and edges for the same. The symmetric $n$-complete $k$-regular cacti on given number of vertices are also enumerated.

ACKNOWLEDGEMENT. The authors are highly thankful to anonymous referees for their constructive suggestions and kind comments.

\section{References}

[1] J. A. Gallian, A dynamic survey of graph labeling, The Electronic Journal of Combinatorics, $\sharp$ DS6, $18: 12$ and 49, (2011).

[2] S. K. Vaidya, D. D. Bantva, Labeling cacti with a condition at distance two, Le Mathematiche, 66 : pp. 29-36, (2011).

[3] D. B. West, Introduction to Graph Theory, Prentice -Hall of India, (2001). 
S. K. Vaidya

Department of Mathematics

Saurashtra University, Rajkot - 360005

Gujarat

INDIA

e-mail : samirkvaidya@yahoo.co.in

and

D. D. Bantva

Department of Mathematics

L. E. College, Morvi - 363642

Gujarat

INDIA

e-mail : devsi.bantva@gmail.com 